\title{
ANDROID APP CODE COVERAGE
}

\author{
Dinu MIHAIL-VĂDUVA \\ Bucharest University of Economic Studies, Romania \\ dinu.mihailvaduva@stud.ase.ro
}

\begin{abstract}
Activities related with software quality evaluation for mobile devices are nowadays confronted with a major outpouring of trends related to outstanding mobile evaluation possibilities. In this paper we use statement code coverage (CC), a software quality metric, in order to discover technical conditions needed to accomplish when we will desire to check and prove that the same pattern of manually testing an Android application was really used, through a beta testing process, by an end user. For this mission we are using the regression analysis. Scientific articles were studied concerning different ways to understand and evaluate calculation of statement code coverage for mobile software application and the relationship between existing methods features and the choice that a developer could have against one or another method to evaluate this quality software metric. Our starting point related with this article, that makes a difference, is motivated by the fact that we use a tool that operates in a black box style, without the need to know the Java Android application's source code. Our quest is orientated in the direction of integrate our practical experiment into an educational laboratory process to a better learning of programming and testing software applications for mobile devices.
\end{abstract}

Keywords: mobile applications, mobile learning, Android, quality metrics, econometrics

JEL Classification: I25, C88, L86

DOI: $10.12948 / \mathrm{ie} 2019.06 .02$

\section{Introduction}

The code coverage is a measure in computer science that embraces a percentage of code that could by touched when a specific software test case was done. Java code coverage tools require technologies that insert statements to app's Java source code and afterward recompilation plus instrumentation to the byte code. Automatic testing concerning multiple versions of an Android application versus a single version of the same Android Application started a research finished by Quoan [1] from which we could see that a major component of their framework was a code coverage generator that inserts automatically instrumentation for every block in byte code level tested in such a way that logs generated exposed which block is executed or not. A recent study realized by Pilgun [2] reveals that code coverage of an Android application is an important metric to evaluate efficiency which is a key attribute regarding software quality evaluation. The main distinction between Pilgun's research and the general trend of other software that covers code coverage inspection was that his method involves a black-box approach with no need to access Android app's code source. Pilgun's tool repository was published [3] and could be implemented by anyone using a software resource that implies Python, Android SDK, and Java to investigate the smali content of any code coverage through any Android's apk package regarding the classes, methods and instructions. This research team [4] reports a percentage of $96.9 \%$ apps successfully instrumented with ACVTool with a small or not noticeable time overhead. Our research helps readers to investigate code coverage of their Android applications 
knowing that a similar goal is a path to a better efficiency regarding time consumption when developers build an Android project.

This paper is structured as follows. The first section shows the salient facts of software quality evaluation about the statement code coverage metric which will be studied in our research. The second section reveals our efforts to extract from scientific literature technical details about the statement code coverage evaluation, dedicated frameworks to measure it. The third section shows a methodology to investigate, with ACVTool, an evaluation about a quality percentage regarding statement code coverage for our own Android application [5], virtually adapted, using Java language. In this section we put a special accent toward ACVTool architecture. This section explains technical considerations and implications about the process of checking manually beta testing of an Android application binary file (apk), with an emulator available into Android SDK, from the standpoint concerning automatically evaluation process of the statement code coverage. The fourth section explains our proposed solution to check and prove with the help of a statement code coverage percentage and how to recognize with a general classification a pattern in doing a repetitive manual beta testing evaluation, with the help of the Android's Studio SDK emulator. In the fifth section we show our conclusion and future work related to other intentions regarding similar projects.

\section{Literature review}

A research to calculate code coverage using black box and white box techniques during dynamic tests over Android applications was completed by Horvath [6] which underpins the idea that a synergetic effect will be obtained if we follow a central position between them. They emphasize that a black box technique answers to the question what is the result of an application when an input is assigned to it, and the white box answers to the question how the previous result is obtained. This package of techniques returns a code coverage criterion and it offers an important feedback on the quality of the software. Their results regarding code coverage was studied following a path through test cases, redundant code and traceability calculations. The subject of the user's point of view about the necessity to search quality software metric using code coverage reports, through Android mobile developers, was a key target for scientific research generated by [7] which deploys a survey regarding code coverage issues, automated testing opportunities and test cases. This survey was harbored using the online platform named Qualtrics and the respondents were involved using email. The final outcome from this study was orientated through building of a body of knowledge which will be considered an important asset to any researcher or developer in this field. A study about the process of obtaining code coverage for Android dynamic analysis tools was realized by [8] which disclose their approach as a set of connected processes between decompiling an apk file, inserting instrumentation code, recompiling, repackaging and testing the final product modified. They report a low rate of successfully repackaging Android apk file, situated at the value of only $36 \%$, but the code coverage results were similar to those prefabricated with EMMA tool. EMMA [9] is compatible with all Android devices with version 4.0.3.or higher and it is recognized as a tool for Java code coverage. In no way connected to the static analysis previously mentioned they started a new approach using offline tools like Android emulator and DroidBox to determine code coverage on the same apk packages used in static analysis. DroidBox [10] is a software tool to build dynamic analysis of Android applications which is known by the fact that two graphs could be generated automatically. First graph is related to the temporal sequences of the operations and the second one is presented using a tree map layout that offers parallel similarity between package actions. They reported similar results between offline and online tools but some observations were influenced by the UI operations commonly encountered like login activity, license messages or pop-up ads. Online tools used in this study like Anubis, 
TraceDroid and CopperDroid are examples of discontinued software products which cannot be used. A study about the quality of industrial applications regarding code coverage and fault detection under examination of Google's tool named Monkey [11] was performed by [12] to identify user interface behavior and consequences related to it, reproduced by human gestures, under an assault of randomly user interface streams events sequences. The Monkey tool from Google returns the highest method coverage on 22 of 41 Android applications whose method coverage was obtained. This result was an answer to the question regarding what is the code coverage obtained by a test generation tool over an industrial Android application. Monkey tool can be used as a stress - test software in a random style and is embedded into emulator or device where it runs. Other test generation tool selected for this study was DroidBot [13] which allows developers to design scripts for a personalized strategy exploration. DroidBot is a test generator input for Android application that generates a transition graph after testing. The main characteristics about DroidBot convey to the fact that it does not require application instrumentation, could produce user interface structures for further analysis, events are not based on a random model but a GUI one and it is programmable. For code coverage measurement they used a tool named Ella [14] to perform collecting statistics of method coverage. Ella tool is designed to instrument Android applications to record which methods were being touched with time-stamped value registered or estimated values of parameters of methods. This tool requires Unix operating system, Android SDK and Java SDK. Another study about measuring code coverage using EMMA was done by a team [15] which shows that their technique requires an injection of a method call during the application testing process and the EMMA tool was launched by an instrumentation activity responsible with starting of the main activity of the every Android application studied. They proposed a software solution, Dynodroid, that is an input generation system for Android applications with a technique constructed around the cycle defined by the sequence observe - execute - select. Their algorithm establishes which events are important to the application in a predefined stage and then select and execute one of these events for the purpose of obtaining a new state where the whole cycle is repeated in the same way mentioned through above sequence. They recognized the importance of the human arbitrary actions when we need an activity, for experimenting some of the Android application functionalities like entering passwords or choosing different settings values, that generate a pause into Dynodroid generating mechanism but resume it immediately after the user's choice is realized.

\section{Methodology}

We have developed our previous Android application [5] and let's say that we are using two teams for our research, one consisting of programming developers, dubbed here with A label, and the other one, named with B label, for beta testing process. Our research question is the following: How do we know if an improvement of a predefined class from our Java's source code, completed by a user from A-Team, is properly manually tested by B-Team, according with an imposed path of sequences, through a manually beta testing process? The answer of this question is linked with a proper classification of code coverage recorded during white box development of the Android application.

Using an adequate classification, from white box's point of view, we could establish a framework for a technical explanation about the flow of sequences really tested by an end user, in charge with a beta testing duty, against the primary objective required by the A-Team. For understanding our study, we propose a generic and general model for a beta testing manually process of a predefined class, embedded into an Android application, that could be seen as a set of predefined paths, required to be tested by A-Team, like those depicted into figure 1 


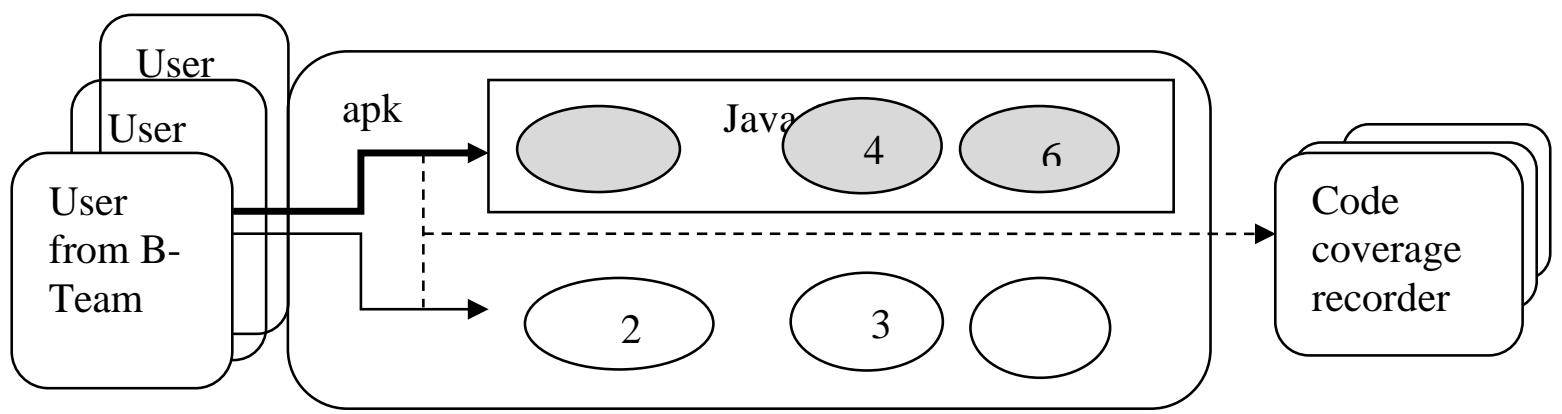

Figure 1. The flow sequence requested by A-Team for beta testing process required to be used by those users from B-Team

Using figure 1 we try to explain that a group of different users, from B-Team, will be asked to execute a beta testing process, only following by the path defined by the flow sequence 1-4-6 and, specifically, will not to be tested using the flow 2-3-5. It is obvious that if above requirement will not be accomplished, during the phase of beta testing, then the effort done to any improvement of the Java class 1 from figure 1 could not be seen by the any code coverage software recorder. On the contrary a high score of statement code coverage recorded during " $i$ " beta testing process should be interpreted as an indicator that the flow sequence 1-4-6 asked from A-Team was completed normally. It is important to say that the A-Team is concerned only to improve the Java's class, shown in figure 1, named with "Java 1", and this will be, for our above research question, predefined class needed to be improved. The model applied to those users from B-Team will be considered as a black box one, where we know what is the result, and the model applied to the A-Team will be considered as a white box, from which we know what the cause regarding of the result is obtained by a black box model. We know from [6] that merging of these two models should obtain a synergetic effect concerning the quality of the software.

In order to evaluate statement code coverage we are using a recorder previously developed by a research team [2]. The ACVTool [2] is capable to establish a measure of the level concerning statements, methods and classes executed or touched during a dynamic testing applied of an Android Java application, without knowing its source code, known by the name of the statement code coverage. This method generates detailed description supported by HTML and XML format from which we obtain tree level reports viewing expanded or collapsed packages, through inner classes, methods and instructions, presented by subsequently numerical calculations when instrumentation was completed. The architecture of ACVTool is represented by three levels.

First level shows that original apk suffers a modification generated by some byte code probes injected into a smali code version of the apk original file. This smali code version of the original apk file was automatically obtained during a previous disassembling process supported by backsmali disassemble [16] integrated into apk tool. After this process a new one is automatically executed meaning a repackaging of the whole content into a new apk file with the help of the apksigner. The last tool, apksigner, is located under Android Studio SDK and is used to properly sign the apk file to confirm that apk's signature will be verified successfully on all versions of Android platforms. This level generates an instrumentation report that will be combined with runtime report produced after the third level into a file with pickle extension. The second level shows that modified apk is installed into an emulator or a physical device 
and, afterward, a process of initiating instrumentation is launched.

The third level shows that ACVTool initiate a drag operation of a runtime report from external memory of the device, used in previous phase, where it was recorded during Android application testing. This report is overlapped with the instrumentation report generated by the first level. This report offers a sum of information starting with the name of the smali package followed by a percentage number indicating the ratio of the code coverage and numbers representing missed versus executed or touched instructions along the process of testing the Android application.

Our research methodology was cyclically developed using a new architecture illustrated in figure 2 .

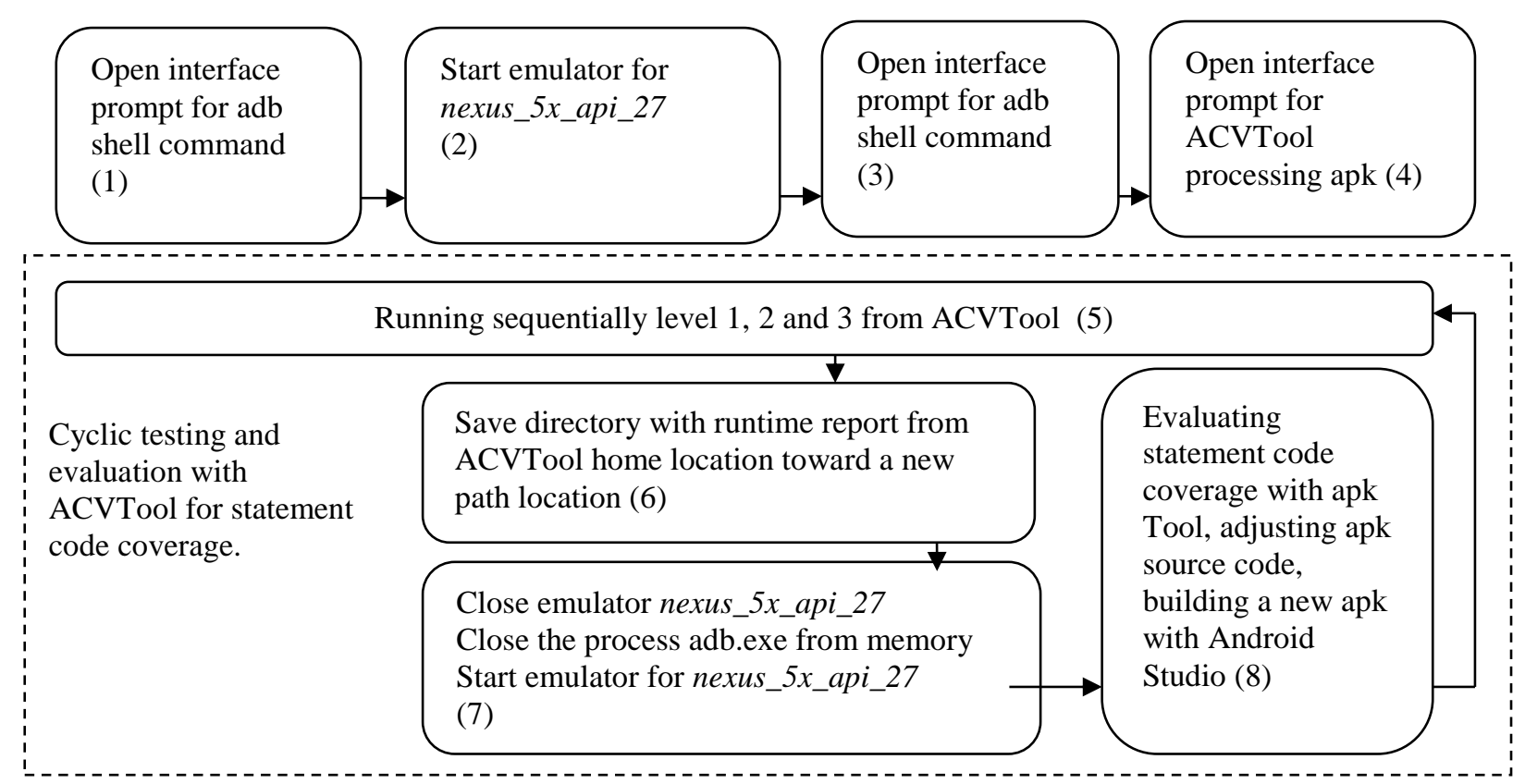

Figure 2. The methodology used to study in our research the statement code coverage

The research team [2] establishes a performance of ACVTool publishing a value of $96.7 \%$ healthy instrumented apps with an average total instrumentation time situated at the value of 36.2 seconds per application.

\section{The proposed solution}

For our study we choose to apply ACVTool to our previous Android Java application [5] to evaluate the efficiency of the statement code coverage metric when we want to classify the activity of beta testing accomplished by a group of users. We proceed for collecting values concerning statement code coverage with ACVTool to find a general classification of statement code coverage established during white box's development. Our software resources allocated to this study are represented by Android Studio SDK from which we are using the adb.exe (Android Debug Bridge). In this case, the emulator used in our experiment is nexus_5x_api_27. Our operating system was Windows 10 . We used three interfaces to operate with adb tool, ACVTool and the last one used for starting the Android emulator. There is necessary to say that testing for our Android Java apk project was conducted in a manual style and Android Studio was not loaded during this methodology. We choose a manual apk testing style because in our research we want to evaluate any modification with regression analysis regarding the correlation between dates recorded with ACVTool when the team in charge with testing 
Android apk application executes different task at the level of the emulator. The stage 6 in figure 2 is necessary because any new testing and evaluation for statement code coverage with ACVTool overwrite the local default directory used to save the final HTML and XML runtime report. Our study refers to the general framing of different use cases, regarding statement code coverage, at the level of using our Android application into emulator, presented into the figure 3.

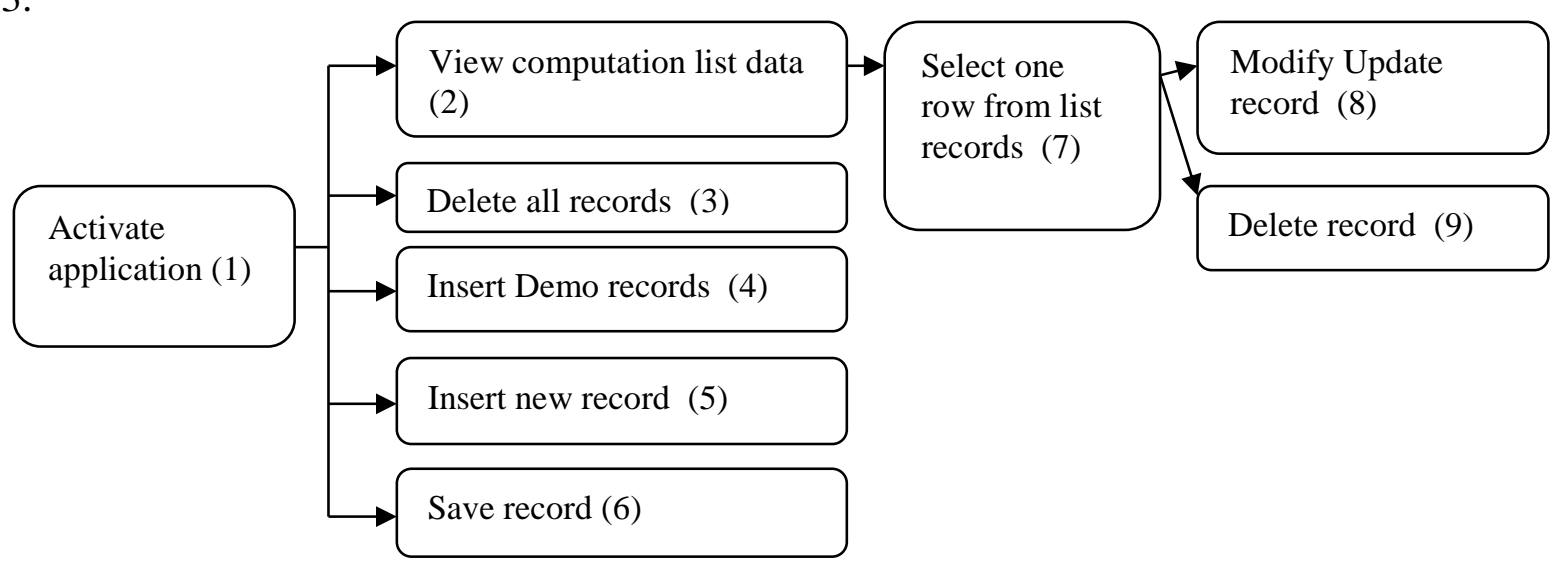

Figure 3. The cases used for flow sequence applied to beta testing process

The target class DatabaseProject and it was monitored using ACVTool. Our experimental data, from white box's point of view, are classified in table 1.

Table 1. Data obtained related to statement code coverage white box use cases

\begin{tabular}{|l|l|l|l|}
\hline Interval & $\begin{array}{l}\text { Minimum } \\
\text { Value CC }\end{array}$ & $\begin{array}{l}\text { Maximum } \\
\text { Value CC }\end{array}$ & Flow Sequence \\
\hline 1 & 0 & 9.69 & Activate application's interface (1) \\
\hline 2 & 11.47 & 15.67 & Insert + Save + Delete all records (1-5-6-3) \\
\hline 3 & 15.67 & 17.44 & Insert + Save (1-5) \\
\hline 4 & 17.44 & 35.7 & Insert database Demo (1-4) \\
\hline 5 & 35.7 & 37.47 & View computational list (1-2) \\
\hline 6 & 37.47 & 39.25 & View computational list + Select on row (1-2-7) \\
\hline 7 & 39.25 & 40.87 & $\begin{array}{l}\text { Insert database Demo + Insert + Save + Delete all Records } \\
(1-4-5-6-3)\end{array}$ \\
\hline 8 & 47.33 & 67.37 & Insert database Demo + Update + Delete (1-4-2-7-8-9) \\
\hline 9 & 83.36 & 96.93 & $\begin{array}{l}\text { Insert database Demo + Update + Delete + Save new } \\
\text { records (1-4-2-7-8-9-6) }\end{array}$ \\
\hline
\end{tabular}

From black box's point of view, a group of user in charge with beta testing duties uses the Android application instrumented with AVCTool and loaded into an emulator and obtained the values recorded into the table 2 .

Table 2. Data obtained related to statement code coverage

\begin{tabular}{|c|c|c|c|c|c|c|c|c|}
\hline User & $\begin{array}{l}\text { Code } \\
\text { Coverage } \\
\text { (CC) (\%) }\end{array}$ & $\begin{array}{l}\text { Missed } \\
\text { Line }\end{array}$ & Line & $\begin{array}{l}\text { Missed } \\
\text { Method }\end{array}$ & Method & $\begin{array}{l}\text { Missed } \\
\text { Class }\end{array}$ & $\begin{array}{l}\text { Clas } \\
\text { s }\end{array}$ & $\begin{array}{l}\text { Interval } \\
\text { Classification for } \\
\text { beta testing }\end{array}$ \\
\hline 1 & 39.25 & 376 & 619 & 12 & 19 & 0 & 1 & 6 \\
\hline 2 & 40.87 & 366 & 619 & 12 & 19 & 0 & 1 & 7 \\
\hline 3 & 67.37 & 202 & 619 & 6 & 19 & 0 & 1 & 8 \\
\hline 4 & 95.15 & 30 & 619 & 3 & 19 & 0 & 1 & 9 \\
\hline 5 & 35.7 & 398 & 619 & 12 & 19 & 0 & 1 & 5 \\
\hline
\end{tabular}




\begin{tabular}{|l|l|l|l|l|l|l|l|l|}
\hline 6 & 39.25 & 376 & 619 & 12 & 19 & 0 & 1 & 6 \\
\hline 7 & 67.37 & 202 & 619 & 6 & 19 & 0 & 1 & 8 \\
\hline
\end{tabular}

Using general classification shown into the table 1 we consider the following outcomes classified after the code coverage obtained. In this way we have a technical disclosure about the activity deployed by any end user in charge with beta testing activity.

The formula used to calculate the values, allocated to statement code coverage, indicated by code coverage $(\mathrm{CC})$, in Table 1 is:

$$
\text { Statement code coverage }(\mathrm{CC})=100-\frac{\text { Missed Line }}{\text { Line }} \times 100(1)
$$

From table 2 we concluded that only user with number 4 , with $95.15 \%$ code coverage (CC), has reached the flow sequence asked for a complete beta testing required. All statement code coverages shown in table 1 are reproducible results. The highest value obtained for the statement code coverage is assigned to the exhaustive way of doing a beta testing process against an Android application. The flow of sequence studied by a beta testing process will have the statement code coverage established by the highest code coverage embedded into the primary sequences. Testing the composite elements of a flow sequence will not have a meaning that all statement code coverage allocated to every element will be added to the final result. If an end user in charge with beta testing repeat execution for a function, then this fact does not mean a highest code coverage result. In this case the code coverage result is determined by the use case specified by the function itself. The scenario used to manually test the apk application has a starting point during the second level indicated under the fifth label in figure 2. After installation of instrumented apk into emulator and starting it we proceed to use and test the Android apk application using only a pattern of actions preselected.

We proposed that arithmetic mean formula for overall statement code coverage allocated to the Java sources of the Android application apk should be calculated without taking into consideration values reported for Android support classes, $\mathrm{R}$ classes or other classes automatically generated and always embedded into any final apk product according with the latest Android technology production.

\section{Conclusions and future work}

Technical conditions discovered, seen as a software perspective, for a valid implementation of ACVTool, toward a beta testing process of Android applications, using code coverage metric, will be correlated with a proper utilization of Android operating system, Android Debug Bridge to consolidate the path between a mobile emulator and ACVTool, Java and Python. There are real opportunities to track the evolution of different beta testing scenarios, inside of a specialized educational laboratory for mobile programming, when we try to manually testing an Android application. The information recorded into runtime report generated by ACVTool could be automatically extracted and inserted into a database log file to a future interpretation. For this objective there is a need to use SQLite with the help of Android Debug Bridge. The statement code coverage is a software quality metric that could be rapidly evaluated and generates feedback actions to improve general structure of an Android's application and is an excellent topic for a laboratory dedicated to study programming and testing Android applications for mobile devices. The following directions will be considered to improve our work:

- Searching similar trends to evaluate statement code coverage.

- Studying the correlation between internal resources, of a mobile device, used by an Android application and the code coverage metric. 
www.conferenceie.ase.ro

\section{References}

[1] D. Quoan, Y. Guowei, C. Meiru, H. Darren and R. Jefferson, "Redroid: A Regression Test Selection Approach for Android Applications," in Proceedings of the International Conference on Mobile Software Engineering and Systems, Austin, Texas, USA, 2016.

[2] A. Pilgun, O. Gadyatskaya, S. Dashevskyi, Y. Zhauniarovich and A. Kushniarou, "DEMO: An Effective Android Code Coverage Tool," in Proceedings of the 2018 ACM SIGSAC Conference on Computer and Communications Security, Toronto, Canada - October 15 - 19, Pages 2189-2191, 2018.

[3] A. Pilgun, "pilgun/acvtool," Oct 2018. [Online]. Available: https://github.com/pilgun/acvtool. [Accessed 9 March 2019].

[4] A. Pilgun, O. Gadyatskaya, S. Dashevskyi, Y. Zhauniarovich and A. Kushniarou, "Finegrained Code Coverage Measurement in Automated Black-box Android Testing," eprint arXiv:1812.10729, December 2018.

[5] D. Mihail-Vaduva, "Enriching Curricula with Mobile Solutions," Informatica Economică, vol. 22 , no. $3,2018$.

[6] F. Horváthy, S. Bognáry, T. Gergelyy and R. Ráczy, "Code Coverage Measurement Framework for Android Devices," Acta Cybernetica 21, p. 439-458, 2014.

[7] M. Linares-Vasquez, C. Bernal-Cardenas, K. Moran and D. Poshyvanyk, "How do Developers Test Android Applications ?," in International Conference on Software Maintenance and Evolution ( ICSME ), Shanghai, China, 17-22 Sept., 2017.

[8] C.-Y. Huang, C.-H. Chiu, C.-H. Lin and H.-W. Tzeng, "Code Coverage Measurement for Android Dynamic Analysis Tools," in IEEE International Conference on Mobile Services, New York, NY, USA, 27 June-2 July, 2015.

[9] "EMMA: a free Java code coverage tool," [Online]. Available: http://emma.sourceforge.net/. [Accessed 19 March 2019].

[10] P. Lantz, "pjlantz/droidbox," [Online]. Available: https://github.com/pjlantz/droidbox. [Accessed 19 March 2019].

[11] "UI/Application exerciser Monkey," [Online]. Available: http://developer.android.com/tools/help/monkey.html. [Accessed 19 March 2019].

[12] W. Wang, D. Li, W. Yang, Y. Cao, Z. Zhang, Y. Deng and T. Xie, "An Empirical Study of Android Test Generation Tools in Industrial Cases," in ACM/IEEE International Conference on Automated Software Engineering, Montpellier, France - September 03 $07,2018$.

[13] "honeynet/droidbot," [Online]. Available: https://github.com/honeynet/droidbot. [Accessed 19 March 2019].

[14] "ELLA: A Tool for Binary Instrumentation of Android Apps," [Online]. Available: https://github.com/saswatanand/ella. [Accessed 3 March 2019].

[15] A. MacHiry, R. Tahiliani and M. Naik, "Dynodroid: An Input Generation System for Android Apps," in Joint Meeting on Foundations of Software Engineering, Saint Petersburg, Russia - August 18 - 26, 2013.

[16] JesusFreke, "smali/backsmali. 2018.," JesusFreke/smali, 2018. [Online]. Available: https://github.com/JesusFreke/smali. [Accessed 10 March 2019]. 